\title{
We thank those who have given their time to referee for Diabetologia, all of whom are listed on our website. Special thanks are due to our key referees, listed below, who have each provided at least three timely and well-rated reports over the past year.
}

\author{
Ahrén B; Lund \\ Amiel SA; London \\ Badenhoop K; Frankfurt \\ Bailey CJ; Birmingham \\ Bain S; Birmingham \\ Balkau B; Villejuif \\ Barroso I; Cambridge \\ Beck-Nielsen H; Odense \\ Bilous RW; Middlesbrough \\ Böhm B; Ulm \\ Boitard C; Paris \\ Bolli G; Perugia \\ Bonifacio E; Milan \\ Bonner-Weir S; Boston \\ Borch-Johnsen K; Copenhagen \\ Bouwens L; Brussels \\ Calcutt N; La Jolla, CA \\ Ceriello A; Udine \\ Chaturvedi N; London \\ Christie MR; London \\ Clark MG; Hobart \\ Coppack SW; London \\ Cotter M; Aberdeen \\ Cryer PE; St Louis \\ Cull C; Oxford \\ Dekker JH; Amsterdam \\ Dornhorst A; London \\ Dunger DB; Cambridge \\ Eckel J; Düsseldorf \\ Edmonds ME; London \\ Eizirik DL; Brussels \\ Eriksson JW; Umeå \\ Fasshauer M; Leipzig \\ Feldt-Rasmussen B; Copenhagen \\ Fernandez-Real JM; Girona \\ Ferrannini E; Pisa \\ Ferrer J; Barcelona \\ Feskens EJM; Bilthoven \\ Flatt P; Coleraine \\ Flyvbjerg A; Aarhus \\ Forrester J; Aberdeen \\ Frayling TM; Exeter \\ Froguel P; Lille \\ Gauguier D; Oxford \\ Gloyn AL; Oxford \\ Gnudi L; London \\ Goodpaster BH; Pittsburgh \\ Green A; Aarhus \\ Green IC; Brighton \\ Grill V; Trondheim \\ Groop L; Malmö \\ Gylfe E; Uppsala \\ Halban P; Geneva
}

Hansen T; Gentofte

Häring H-U; Tübingen

Hattersley AT; Exeter

Hermans MP; Oxford

Herrera P; Geneva

Hitman GA; London

Holly J; Bristol

Holthöfer H; Helsinki

Home PD; Newcastle upon Tyne

In't Veld P; Brussels

Jeremy JY; Bristol

Jonas JC; Brussels

Kadowaki T; Tokyo

Karlsen AE; Gentofte

Karpe F; Oxford

Klaus S; Potsdam-Rehbrücke

Knip M; Helsinki

Kolb H; Düsseldorf

Krentz AJ; Southampton

Kyvik KO; Odense

Laakso M; Kuopio

Landgraf R; Munich

Leech N; Newcastle upon Tyne

Lenzen S; Hannover

Leslie RD; London

Lévy-Marchal C; Paris

Lindsay R; Glasgow

Lindström J; Helsinki

Maassen JA; Leiden

MacDonald IA; Nottingham

Malecki M; Krakow

Malik RA; Manchester

Mann JI; Dunedin

Marette A; Ste Foy

Marshall S; Newcastle upon Tyne

McCance DR; Belfast

McKinney PA; Leeds

Meigs J; Boston

Mingrone G; Rome

Mogensen CE; Aarhus

Morgan NG; Plymouth

Mühlhauser I; Hamburg

Nauck M; Bad Lauterberg

Orchard T; Pittsburgh

Otonkoski T; Helsinki

Parving HH; Gentofte

Paschke R; Leipzig

Patterson CC; Belfast

Pedersen O; Gentofte

Pickup J; London

Pieber TR; Graz

Pinkney J; Liverpool

Pociot F; Gentofte
Porta M; Turin

Pozzilli P; Rome

Remacle C; Louvain-la-Neuve

Ridderstråle M; Malmö

Roep BO; Leiden

Rorsman P; Lund

Rutter G; Bristol

Sandler S; Uppsala

Sauerwein HP; Amsterdam

Scharfman R; Paris

Scherbaum WA; Düsseldorf

Schmitz O; Aarhus

Scott FW; Ontario

Shore A; Exeter

Smith R; Bristol

Smith U; Göteborg

Soria B; Alicante

Spranger J; Bergholz-Rehbrücke

Stehouwer CDA; Amsterdam

Stene LC; Oslo

Stumvoll M; Leipzig

Sugden MC; London

Sundkvist G; Malmö

Tarnow L; Gentofte

Taskinen MR; Helsinki

Tavare J; Bristol

Taylor R; Newcastle upon Tyne

Tesfaye S; Sheffield

Thorens B; Lausanne

Tiedge M; Hannover

Tomkin G; Dublin

Tuomi T; Helsinki

Undlien DE; Oslo

Viberti G; London

Vidal-Puig A; Cambridge

Wahren J; Stockholm

Walker J; Edinburgh

Walker M; Newcastle upon Tyne

Wareham N; Cambridge

Watts GF; Perth

Weir GC; Boston

Welsh M; Uppsala

Welsh N; Uppsala

Wilkin TJ; Plymouth

Williams AJK; Bristol

Williams DRR; Swansea

Wong S; Bristol

Yki-Järvinen H; Helsinki

Zeggini E; Oxford

Zerbini G; Milan

Ziegler AG; Munich

Zierath JR; Stockholm

Zorzano A; Barcelona 